\title{
The Effects of Combustion on Turbulent Statistics in a Supersonic Turbulent Jet
}

\author{
Yaowei $\mathrm{Fu}^{1,2}$, Changping $\mathrm{Yu}^{1,2}$, Zheng $\mathrm{Yan}^{1,2}$ and Xinliang $\mathrm{Li}^{1,2 *}$ \\ ${ }^{1}$ LHD, Institute of Mechanics, Chinese Academy of Sciences, Beijing 100190, China \\ 2 School of Engineering Science, University of Chinese Academy of Sciences, \\ Beijing 100049, China
}

Received 13 August 2018; Accepted (in revised version) 6 December 2018

\begin{abstract}
The effects of combustion on turbulent statistics in a supersonic turbulent jet are investigated by using direct numerical simulation (DNS). To study the combustion effects, two DNS cases (reacting case and non-reacting case) are conducted and the comparison of turbulent statistics are used to study the combustion effects. The medium of jet is $85 \%$ hydrogen with temperature $305 \mathrm{~K}$ and the co-flow is hot air with temperature $1150 \mathrm{~K}$. The Reynolds numbers based on the jet diameters are 22000 and the jet Mach numbers are 1.2. The DNS results show that combustion case has both larger decay rate constant of centerline streamwise mean velocity and larger spreading rate of half-velocity jet width than the non-reacting case. It also shows that the combustion case achieves the self-similarity slower. For reacting jet, the vortex structures are more smooth in the near field and vortex filament structures are larger in the far field. Combustion causes the turbulent zone to be delayed downstream and increases the probability of strong velocity pulsation.
\end{abstract}

AMS subject classifications: 76F06, 76J20, 76V05

Key words: Supersonic jet, turbulent combustion, direct numerical simulation.

\section{Introduction}

The urgent need for hypersonic vehicles has promoted the development of scramjet. In terms of scramjet, one of the most significant characteristics is the supersonic turbulent combustion. In a study of 1958, Weber and MacKay [1] demonstrate that combustion can take place in supersonic airflows. However, until 2004, the successful flight of the $X-43$ represents the first successful attempt to fly a vehicle that included an integrated

${ }^{*}$ Corresponding author.

Emails: fuyaowei@imech.ac.cn (Y. W. Fu), cpyu@imech.ac.cn (C. P. Yu), yanzheng@imech.ac.cn (Z. Yan), lixl@imech.ac.cn (X. L. Li) 
hypersonic airframe-scramjet engine [2]. The primary reason of the very slow development of the scramjet is the complicated interaction between combustion and turbulence. Combustion has different effects on turbulence. It can enhance turbulence and can also weaken turbulence. The expansion of fluid strengthens turbulence and the increase of viscosity due to higher temperature weakens turbulence. In addition, turbulence itself is also an extremely complex phenomenon in non-reaction fluid mechanics involving various time scales and length scales [3]. Therefore, it's necessary to explore the turbulent characteristics under combustion.

The physical processes in the supersonic combustion chamber are more intricate than in most of the other propulsion systems because of the short residence time, the large gradients of species and velocities, the closely coupled interaction between mixing and chemical kinetics, the very high temperature so that the real gas effects have to be considered. Therefore, there is few experimental data that can be found in literatures on supersonic combustion due to difficulties in measuring techniques and simulating highenthalpy conditions in ground-based combustion facilities [4]. Although flight tests are highly effective, they are incredibly expensive so that no country or organization can afford them forever. In addition, because of the extremely complicated form of the terms with respect to the chemical reaction rate and heat release rate in the conservation equations of species and energy, the approach of the theoretical analysis is almost impossible. Thanks to the fast advancement of the computer technologies and the numerical theories, computational fluid dynamics (CFD) methods, including direct numerical simulation (DNS), large eddy simulation (LES), Reynolds-averaged Navier-Stokes equations (RANS), have become a promising and necessary method to understand the intrinsic characteristics of the turbulent reacting flow [5].

Turbulent jet, because of its wide application in engineering, has been studied in detail. The ultimate aim of these efforts is to understand the mechanism of the turbulent jet and then to control it. Papamoschou [6] focuses his attention on the growth rate and turbulent structures of the compressible shear layer. By analyzing his experimental data with free stream Mach number ranging from 0.2 to 4 , he defines a compressibilityeffect parameter in which the convective Mach number is used. But in some extreme conditions, the existence of the convective Mach number is inappropriate. For solving this problem, Slessor [7] proposes a new shear layer growth rate compressibility-scaling parameter. Using the method of DNS for round jets with different inflow conditions, Boersma [8] presents that the universal self-similarity for the free jet is nonexistent and correct scaling is needed for different experimental and numerical data. Bogey [9] uses LES method to study the influences of different Reynolds numbers on jet self-similarity and turbulent features. Numerical results demonstrate that the higher Reynolds number can make the jet flow achieving self-similarity slower. However, the turbulent features do not depend significantly on the Reynolds number. Rao $[10,11]$ experimentally and numerically investigates the effects of nozzles of complex geometry on supersonic free jets with Mach number 1.8. His research shows that complex nozzles are beneficial to mixing enhancement and the centerline axial velocities for different nozzles are all satisfied with 